\title{
Sleep pattern in a group of patients undergoing hemodialysis compared to control
}

\author{
Reem El Sayed Hashem ${ }^{* *}$, Tarek Asaad Abdo ${ }^{1}$, Iman Ibrahim Sarhan² and Amr Mohammed Mansour ${ }^{2}$
}

\begin{abstract}
Background: Patients with chronic kidney disease progress regularly every year to end-stage renal disease and have to undergo dialysis. Sleep disturbances have been reported to be frequent among patients receiving dialysis and contributing to the increase of their mortality and morbidity. The present research aimed to study the sleep pattern in hemodialysis patients and the risk factors associated. This cross-sectional case-control study included 40 subjects divided into 2 groups: 20 cases recruited from Ain Shams University Hospital's dialysis unit and 20 in the control group with normal Pittsburgh Sleep Quality Index score matched for age and sex. Both groups were subjected to overnight polysomnography, and the cases group was assessed by the Pittsburgh Sleep Quality Index to determine their sleep quality.

Results: Nearly all polysomnographic parameters were significantly abnormal in the cases group except for sleep onset latency $(P>0.05)$, showing obstructive sleep apnea and periodic limb movement ( $P$ value 0.001). Based on their Pittsburgh Sleep Quality Index score, 30\% were classified as good sleepers and 70\% as bad sleepers. On comparing both groups, a significant difference was found. Poor sleepers had more worse sleep efficiency (62.9\%), spent longer time during their sleep in stage 1 (26.6\%) with shorter REM onset latency (113.5 \pm 99.5$)$, and had a longer duration of illness with lower serum creatinine level compared to good sleepers.

Conclusions: The prevalence of obstructive sleep apnea and periodic limb movement in hemodialysis patients is high; patients with longer time on dialysis are at more risk of sleep disorders, whereas hemoglobin levels, BUN, and other demographic factors do not seem to play a role in sleep disorder. Hence, patients on hemodialysis need to be screened for sleep disorders so as to improve their mortality and morbidity.
\end{abstract}

Keywords: Sleep, Hemodialysis, Pattern, Obstructive sleep apnea

\section{Background}

End-stage renal disease (ESRD) corresponds to a glomerular filtration rate (eGFR) of $<15 \mathrm{~mL} / \mathrm{min} / 1.73 \mathrm{~m}$ [1]. It showed variable-elevated incidence across the world where in the USA, the annual incidence rate is 355 per million [2]. In Europe, it reaches 135,000 per year [3]. Saudi Arabia reports a prevalence rate of 5.7\% [4]. According to the 9th Annual Report of The Egyptian

\footnotetext{
*Correspondence: dr.reemhashem@gmail.com

${ }^{1}$ Okasha Institute of Psychiatry, Faculty of Medicine, Ain Shams University,

Cairo, Egypt

Full list of author information is available at the end of the article
}

Renal Registry, the prevalence in Egypt raised to 483 patients per million [5].

At this stage, survival and quality of life are sustained by kidney replacement therapy, which includes hemodialysis (HD), peritoneal dialysis, and kidney transplantation [6]. Yet, most patients are treated with dialysis due to the scarcity of donor organs and contraindications to transplantation $[7,8]$.

A global survey was done on nephrologists in over 90 countries to assess the reimbursement for dialysis, suggesting the number of patients receiving HD worldwide was approximately $2,600,000$ patients [6] and is expected to be doubled to 5.4 million by 2030 [9]. Hence, HD 
became the main and most widely used replacement treatment for ESRD patients.

With advances in dialysis techniques and medical care, mortality and morbidity rates of patients on regular hemodialysis have markedly declined, yet this is not the only goal for those patients further improvement of their quality of life has become the aim of medical practitioners. And in order to achieve that, both physical and mental health needs to be satisfied and maintained. However, many patients on dialysis suffer from sleep disorders which undoubtedly affect both their physical and mental health status [10] and consequently worsen their quality of life, so this problem needs to be addressed.

The progression to ESRD appeared to be correlated with the development of sleep disorders [11]. This can be explained by the ineffective glomerular filtration occurring at this stage leading to an inability to maintain normal homeostasis affecting various metabolic products including vital bioelements and proteins. This dysregulation in homeostasis might impact sleep in various ways [12].

HD patients report sleep problems more than twice as frequently as healthy control subjects [13]. Sleep disturbances, including insomnia, obstructive sleep apnea (OSA), restless legs syndrome, periodic limb movements disorder, and excessive daytime sleepiness, have been frequently reported in those patients, and they are associated with a negative effect on the quality of life and functional status [14].

A large number of data suggest a bidirectional relationship between OSA and CKD. That is, CKD likely confers an increased risk of OSA, which is related to declining kidney function status, being more prevalent in ESRD patients, compared to CKD patients not on dialysis, and OSA may in turn increase the risk of renal injury [15].

The prevalence of obstructive sleep apnea is higher than in the general population, as reported to occur in at least 50 to $60 \%$ of chronic kidney disease patients with ESRD $[16,17]$. It affects them tremendously in various ways as the reduction in the airflow occurring during OSA episode leads to acute derangements in gas exchange and recurrent arousals from sleep. This leads to excessive daytime sleepiness, cognitive impairment, decreased work performance, and fall in health-related quality of life. Also as evidenced by many studies that OSA may contribute to the development of systemic hypertension [18], cardiovascular disease [19], and abnormalities in glucose metabolism [20], as the repeated oxygen saturation drops occurring frequently during sleep, increases oxidative stress, and stimulates the sympathetic nervous system [21].

As a result, OSA may aggravate the medical condition of ESRD patients increasing their mortality rate as proved earlier that cardiovascular disease is the leading cause of death in dialysis patients, and occurrence of sudden death [22] and the presence of diabetes mellitus and poor glycemic control are often associated with an increase of mortality [23, 24]. So, it is not only affecting their quality of life but also poses a threat to their survival, yet it is undiagnosed in many cases.

Hence, the identification and treatment of OSA or other sleep disorders are of clinical significance, as early intervention can diminish daytime fatigue, enhance physical activity, and thus result in improved metabolic control including glycemic control. Diagnosing OSA is therefore important in the management of HD patients, since it is a treatable condition [21].

The purpose of the current study was to explore whether the patients undergoing hemodialysis suffer from sleep disorders, aiming to raise awareness across medical disciplines especially nephrologists and providing them with sufficient knowledge to identify those affected with sleep disorders and implement the treatment in place to enhance their quality of life and improve mortality among those groups of patients by preventing the metabolic derangements caused by OSA.

\section{Methods}

This cross-sectional case-control comparative study was conducted in the dialysis units in the nephrology department and Okasha Institute of Psychiatry in Ain Shams University Hospital over 6 months duration during which a convenient sample of 20 patients was chosen fulfilling the inclusion criteria. The procedure of the study was accepted by the ethical committee of Ain Shams University, Cairo, Egypt.

The study included 40 subjects divided into 2 groups:

Cases group: 20 patients receiving regular hemodialysis 3 sessions/week in the dialysis unit fulfilling the inclusion criteria, diagnosed as end-stage renal failure and on regular hemodialysis for at least 6 months, both sexes, age group 18-50 years and giving written informed consent after the purpose of the study was explained, and were given an opportunity to decline to participate in the study without interfering with their medical care. Patients with a history of psychiatric illness before starting dialysis; receiving sedatives, antipsychotics, and antihistaminic; and with severe heart failure, morbid obesity, or respiratory distress were excluded.

Control group: 20 individuals healthy with no medical or psychiatric illness history taking no medication affecting sleep and with global Pittsburgh Sleep Quality Index score $\leq 5$ matched with the patient group for age and sex were selected from the employees of Okasha Institute of Psychiatry. 


\section{Procedure}

All patients included in the study were subjected to complete the clinical examination with the calculation of body mass index (BMI), serum creatinine and BUN, complete blood count, $\mathrm{Na}$ and $\mathrm{K}$ together with a history of taking about the duration of dialysis, comorbidities, and drug history and assessed for their sleep quality using the Pittsburgh Sleep Quality Index.

Both groups were subjected to the assessment of their sleep pattern using overnight polysomnography (done on the second day of hemodialysis for cases group) in the sleep lab of Okasha Institute of Psychiatry.

\section{Tools used}

\section{Pittsburgh Sleep Quality Index (PSQI)}

This is a self-rated questionnaire, consisting of 19 questions related to sleep quality in the previous month, including estimates of sleep duration and latency, as well as frequency and severity of specific sleep-related problems. The 19 questions are grouped into seven component scores, each weighted equally on a $0-3$ scale. The seven components are summed to yield a global PSQI score (range 0-21); higher scores indicate worse sleep quality [25]. Patients with a global PSQI score $>5$ are conventionally defined as "poor sleepers," whereas those with a score $\leq 5$ are considered "good sleepers." The Arabic version was used which was translated by [26].

\section{Polysomnogram}

The apparatus used is Neurofax EEG2110, Digital Electroencephalograph, Nihon Kohden Corporation, Tokyo, Japan. It can provide immediate staging, scoring, and reporting of the PSG night. Hypnographic all night $8 \mathrm{~h}$ sleep duration PSG provides the following variables:

I. Sleep continuity includes sleep latency, sleep efficiency, and arousal index. Sleep latency: normally about $15 \mathrm{~min}$. Sleep efficiency is usually greater than $90 \%$ in the young and decreases somewhat with age [27]. Arousal index is the total number of arousals per hour of sleep.

II. Analysis of sleep architecture includes percentages of NREM-sleep stages (I, II, III, IV, and SWS) to total sleep time. For the age group 41-50 years, which includes our sample age category the normal $\%$ varies to be stage I $14 \%$, stage II $50 \%$, and SWS $7 \%$ [28].

\section{Periodic Limb Movement Index (PLMI)}

It is the number of muscle contractions occurring per hour of sleep. PLMI of more than 5 and less than 25 is considered mild, PLMI of $>25$ and $<50$ is considered moderate, and $>50$ is considered severe [29].

III. Apneas includes total apnea/hour and desaturation/hour. Apnea-Hypopnea Index (AHI): the total number of complete cessations (apnea) and partial obstructions (hypopnea) of breathing occurring per hour of sleep. AHI can be used to classify the severity of disease as mild 5-15, moderate 15-30, and severe greater than 30 [30].

\section{Respiratory Disturbance Index (RDI)}

This is the average number of episodes of apnea, hypopnea, and respiratory event-related arousal per hour of sleep. The American Academy of Sleep Medicine uses the Respiratory Disturbance Index to determine the severity of obstructive sleep apnea according to the following range: 5-14.9 for mild, 15-29.9 for moderate, and $30+$ for severe [31].

IV. REM sleep analysis includes REM percentage (14\% for ages 41-50 years) [28] and REM sleep latency.

\section{Statistical methods}

Data were analyzed using PASW version 18 (IBM๑) Corp., Armonk, NY, USA). The normality of data was tested using the D'Agostino-Pearson test, and normally distributed numerical variables were presented as mean $\pm S D$. Numerical data were compared using the unpaired Student $t$ test, and qualitative data were compared using the chi-square $t$ test or the Fisher exact test. $P$ value was considered significant if < 0.05 (S). $P$ value was considered non-significant if $>$ 0.1 (NS).

\section{Results}

\section{Demographic and clinical data of cases}

The mean age of the patients was $41.59 \pm 7.12$ years; $13(65 \%)$ were male and most of them were married 16 (80\%). Their BMI was $25.82 \pm 3.05$ which classify them within normal to overweight. The duration of illness was $5.55 \pm 3.5$ years. Ten (50\%) had hypertension while 9 (45\%) had diabetes mellitus. There was no significant statistical difference between cases and control as shown in Table 1. 
Table 1 Socio-demographic characteristics of cases and controls

\begin{tabular}{lllll}
\hline & & Cases & Control & P value \\
\hline Age (years) & & $41.59 \pm 7.12$ & $39.50 \pm 5.41$ & 0.847 \\
Gender & F & $7(35 \%)$ & $7(35 \%)$ & 0.629 \\
& M & $13(65 \%)$ & $13(65 \%)$ & \\
Marital state & Single & $4(20.0 \%)$ & $8(40 \%)$ & 0.150 \\
& Married & $16(80 \%)$ & $12(60 \%)$ & \\
BMI (kg/m $\left.{ }^{2}\right)$ & & $25.82 \pm 3.05$ & $24.32 \pm 2.18$ & 0.083 \\
Duration of illness (years) & $5.55 \pm 3.5$ & & \\
HTN & $10(50 \%)$ & $0(.0 \%)$ & $<\mathbf{0 . 0 0 1}$ \\
DM & $9(45 \%)$ & $0(0 \%)$ & $\mathbf{0 . 0 0 1}$ \\
\hline
\end{tabular}

$B M I$ body mass index, HTN hypertension, DM diabetes mellitus

Table 2 Comparison between cases and control as regards the sleep study findings (polysomnographic variables)

\begin{tabular}{llll}
\hline & Cases & Control & P value \\
\hline Sleep onset latency & $13.3 \pm 8.42$ & $15.6 \pm 2.29$ & 0.213 \\
Sleep efficiency (\%) & $68.13 \pm 12.85$ & $91.35 \pm 1.35$ & $\mathbf{0 . 0 0 0}$ \\
Sleep stage I (\%) & $20.85 \pm 25.41$ & $3.15 \pm .68$ & $\mathbf{0 . 0 0 3}$ \\
Sleep stage II (\%) & $36.76 \pm 14.56$ & $50.33 \pm .43$ & $\mathbf{0 . 0 0 0}$ \\
SWS (\%) & $37.60 \pm 29.75$ & $22.63 \pm .93$ & $\mathbf{0 . 0 3 0}$ \\
REM sleep (\%) & $4.80 \pm 6.02$ & $26.47 \pm 11.40$ & $\mathbf{0 . 0 0 0}$ \\
REM latency (min) & $146.08 \pm 102.62$ & $70.60 \pm 5.75$ & $\mathbf{0 . 0 0 2}$ \\
Arousal index (h) & $32.08 \pm 22.73$ & $.89 \pm 1.03$ & $\mathbf{0 . 0 0 0}$ \\
Sleep total apnea (h) & $9.95 \pm 11.61$ & $.06 \pm .12$ & $\mathbf{0 . 0 0 0}$ \\
Sleep obstructive apnea (h) & $7.23 \pm 9.59$ & $.05 \pm .11$ & $\mathbf{0 . 0 0 2}$ \\
Sleep central apnea (h) & $1.02 \pm 1.38$ & 0 & \\
Mixed apnea (h) & $1.66 \pm 2.24$ & $.01 \pm .02$ & $\mathbf{0 . 0 0 2}$ \\
AHI/h & $13.31 \pm 16.98$ & $.02 \pm .05$ & $\mathbf{0 . 0 0 1}$ \\
Desaturations (h) & $23.95 \pm 22.85$ & $.03 \pm .07$ & $\mathbf{0 . 0 0 0}$ \\
RDI & $23.26 \pm 23.79$ & $.07 \pm .15$ & $\mathbf{0 . 0 0 0}$ \\
PLM Index & $25.37 \pm 31.59$ & $.25 \pm .31$ & $\mathbf{0 . 0 0 1}$ \\
\hline
\end{tabular}

Values are expressed as number (\%) or as mean $\pm S D$, as appropriate $A H I$ apnea-hypopnea index, $R D /$ respiratory disturbance index, $P L M$ Index Periodic Limb Movements Index, REM rapid eye movement sleep, SWS slowwave sleep

\section{Polysomnographic findings in the cases versus control group}

On comparing between both groups, a statistically significant difference was found in almost all sleep parameters assessed by the polysomnography except for sleep onset latency $(P>0.05)$ as shown in Table 2 .

Where the HD patients suffer from less sleep efficiency $(68.13 \pm 12.85)$, they are losing a third of their sleep time. They spend more time in stage I (20\%) and SWS stage (37.60 \pm 29.75$)$ sleep and significantly less time in stage $2(36.76 \pm 14.56)$; they are not getting enough REM sleep $(4.80 \pm 6.02)$ with increased REM latency $(146.08 \pm 102.62 \mathrm{~min})$ and showing frequent arousals (AI; $32.08 \pm 22.73 / \mathrm{h}$ ).

Meanwhile, $65 \%$ of cases experience obstructive sleep apnea (AHI; $13.31 \pm 16.98 / \mathrm{h}$ ) with RDI $23.26 \pm 23.79$ which classify them as having moderate obstructive apnea.

Also, 70\% of patients experience frequent periodic limb movements during the night with PLMI $25.37 \pm 31.59$ categorizing them as periodic limb movements disorder (PLMD) according to the AASM established criterion for PLMD with PLMI cutoff of $\geq 15$ [32] with moderate degree (PLMI $>25<50$ ). Very few central and/or mixed apneas were recorded among the participants.

\section{Quality of sleep among cases}

The patients were classified based on their PSQI score into 2 groups: good sleepers (score less than or equal to 5 ) and poor sleepers (score more than 5). The percentage of good sleepers among our 20 patients was $30 \%$, and bad sleepers were $70 \%$. Then, both groups were compared according to the clinical, demographic, and polysomnographic variables.

Table 3 showed among different variables, only the duration of illness and serum creatinine level showed a significant difference between both groups with $P$ values 0.032 and 0.008 , respectively, where poor sleepers had a longer duration of illness and lower serum creatinine levels.

Regarding polysomnographic variables, there were significant differences between good and poor

Table 3 Comparison between the good and bad sleepers groups as regards demographic and clinical variables

\begin{tabular}{|c|c|c|c|c|}
\hline & & Good sleeper & Poor sleeper & $P$ value \\
\hline Age & & $39.2 \pm 9.2$ & $43.1 \pm 6.0$ & 0.362 \\
\hline \multirow[t]{2}{*}{ Gender } & Female & $2(33.3 \%)$ & $5(35.7 \%)$ & 0.664 \\
\hline & Male & $4(66.7 \%)$ & $9(64.3 \%)$ & \\
\hline \multirow[t]{2}{*}{ Marital state } & Single & $2(33.3 \%)$ & $2(14.3 \%)$ & 0.343 \\
\hline & Married & $4(66.7 \%)$ & $12(85.7 \%)$ & \\
\hline BMI & & $25.1 \pm 1.8$ & $26.1 \pm 3.5$ & 0.413 \\
\hline Duration of illness & & $3.5 \pm 1.9$ & $6.4 \pm 3.7$ & 0.032 \\
\hline HTN & & $1(16.7 \%)$ & $9(64.3 \%)$ & 0.070 \\
\hline DM & & $3(50 \%)$ & $6(42.9 \%)$ & 0.574 \\
\hline Creatinine (mg/dl) & & $12.1 \pm 2.1$ & $8.6 \pm 1.9$ & 0.008 \\
\hline Bun (mg/dl) & & $57.3 \pm 7.1$ & $55.4 \pm 20.6$ & 0.755 \\
\hline $\mathrm{Na}(\mathrm{mEq} / \mathrm{l})$ & & $133.5 \pm 5.8$ & $135.6 \pm 3$ & 0.438 \\
\hline $\mathrm{K}(\mathrm{mEq} / \mathrm{l})$ & & $4.5 \pm .3$ & $4.7 \pm .9$ & 0.517 \\
\hline $\mathrm{Hgb}(\mathrm{g} / \mathrm{dl})$ & & $10.9 \pm 1.2$ & $10.4 \pm 1.3$ & 0.376 \\
\hline WBCs $\left(10^{3} / \mu \mathrm{l}\right)$ & & $7.2 \pm 2.5$ & $7.1 \pm 2$ & 0.930 \\
\hline Platelets $\left(\times 10^{3} / \mu \mathrm{l}\right)$ & & $166.8 \pm 41.6$ & $195.4 \pm 64.6$ & 0.256 \\
\hline
\end{tabular}

$B M I$ body mass index, HTN hypertension, DM diabetes mellitus, $\mathrm{Hg} b$ hemoglobin 
Table 4 Comparison between the good and bad sleepers groups as regards polysomnographic variables

\begin{tabular}{llll}
\hline & Good sleeper & Poor sleeper & P value \\
\hline Sleep onset latency & $8.5 \pm 8.8$ & $15.1 \pm 7.7$ & 0.149 \\
Sleep efficiency (\%) & $80.4 \pm 6.8$ & $62.9 \pm 11.2$ & $\mathbf{0 . 0 0 1}$ \\
Sleep stage I (\%) & $7.5 \pm 7.5$ & $26.6 \pm 28.4$ & $\mathbf{0 . 0 3 3}$ \\
Sleep stage II (\%) & $33.9 \pm 17.6$ & $38.0 \pm 13.6$ & 0.623 \\
SWS (\%) & $51.7 \pm 24.0$ & $31.6 \pm 30.7$ & 0.141 \\
REM sleep (\%) & $6.9 \pm 7.4$ & $3.9 \pm 5.4$ & 0.399 \\
REM latency (min) & $222.0 \pm 66.2$ & $113.5 \pm 99.5$ & $\mathbf{0 . 0 1 2}$ \\
Arousal index (h) & $36.7 \pm 15.6$ & $30.1 \pm 25.5$ & 0.492 \\
Sleep total apnea (h) & $9.8 \pm 8.3$ & $10.0 \pm 13.1$ & 0.962 \\
Sleep obstructive apnea (h) & $5.0 \pm 5.0$ & $8.2 \pm 11.0$ & 0.375 \\
Sleep central apnea (h) & $1.7 \pm 1.5$ & $.8 \pm 1.3$ & 0.240 \\
Mixed apnea (h) & $3.2 \pm 2.8$ & $1.0 \pm 1.7$ & 0.122 \\
SL hypopnea/h & $22.4 \pm 20.1$ & $9.4 \pm 14.5$ & 0.196 \\
Desaturations (h) & $30.0 \pm 24$ & $21.4 \pm 22.7$ & 0.473 \\
RDI & $32.2 \pm 26.1$ & $19.4 \pm 22.6$ & 0.328 \\
PLM Index & $30.7 \pm 23.2$ & $23.1 \pm 35.1$ & 0.576 \\
\hline
\end{tabular}

Values are expressed as number (\%) or as mean $\pm S D$, as appropriate $A H I$ apnea-hypopnea index, $R D I$ respiratory disturbance index, $P L M$ Index Periodic Limb Movements Index, REM rapid eye movement sleep, SWS slowwave sleep

sleepers as regards sleep efficiency with $P$ value 0.001 , stage I percentage $P$ value 0.033 , and REM onset latency $P$ value 0.012 , as poor sleepers have worse sleep efficiency (62.9\%), spending longer time during their sleep in stage $1(26.6 \%)$ which is light sleep with a shorter REM onset latency (113.5 \pm 99.5$)$ min compared to good sleepers, as shown in Table 4.

\section{Discussion}

Sleep-disordered breathing is the most common cause of poor sleep in kidney disease patients with a high prevalence in patients on hemodialysis because of compromised upper airway stability [33, 34]. The morbidity and mortality together with the impairment of the quality of life as a consequence of SDB have intensified the need for making early identification and diagnosis.

In the current study, we undertook PSG in HD patients and controls. We found that obstructive sleep apneas were predominant in HD patients. This finding was in line with $[13,35]$ studies and that of Nicholl and his colleagues' study, whom their data revealed significant increases in the occurrence of obstructive sleep apnea with a prevalence of $57 \%$ in the ESRD group [36]. They also concluded that the high incidence of nocturnal hypoxia in chronic kidney disease with ESRD patients could contribute to both the loss of kidney function and increased cardiovascular risk worsening the patient's condition which confirm the importance of detection.
Owing to this high predominance of OSA, HD patients should be considered from the high-risk groups for developing OSA, and therefore, it is crucial to properly point diagnostic attention to them in the medical settings and screen for their existence.

The reasons for the occurrence of sleep apnea are explained by older age and the presence of other risk factors such as hypertension and diabetes [14]. Also, OSA is estimated to be present more in male $(20 \%)$ than in female (10\%) patients with ESRD [37], which is applied to our sample as most of the cases were males (65\%) belonging to the older age group (41.59 \pm 7.12$)$ having either hypertension $(50 \%)$ or diabetes $(45 \%)$.

PSG revealed the occurrence of periodic limb movements among the cases group with moderate degree corroborating with previous studies stating that it is higher in hemodialyzed patients compared to the general population, and it is often in the moderate-severe range $[38,39]$ and the estimated prevalence is $40-70 \%$ among patients on maintenance dialysis [39-41] compared to $7-8 \%$ in the general population.

Possible etiologies in the development of PLM in ESRD including our patient group appeared to be related to iron deficiency ( $\mathrm{Hgb} 10.9 \pm 1.2 \mathrm{~g} / \mathrm{dl}$ ), the presence of uremia [41], and obstructive sleep apnea conditions [42].

Other PSG findings can be explained by the presence of sleep obstructive apnea and periodic limb movement; the high arousal index $(32.08 \pm 22.73$ vs $16.8 \pm 6.2$ for this age group), the long REM sleep latency (146.08 \pm 102.62 min vs $90 \mathrm{~min}$ ) [43], and the high percentage of the stage S1 sleep (20\% vs 14\%) are as a result of frequent arousals caused by sleep apnea and periodic movement of sleep [43], similarly, the low percentage of stage S2 (36\% vs $50 \%)$ may be explained by sleep fragmentation, obstructive sleep apnea-related arousals, or the increased SWS percentage (37\% vs $7 \%)$. Finally, the reduced REM frequency ( $4 \%$ vs $14 \%$ ) could be explained by BaHammam and his colleagues reporting that REM sleep is reduced in patients with severe OSA [44]. These findings were in concordance with Roumelioti et al. showing similar PSG findings in their HD patients when compared to the CKD and control group [45].

Hence, HD patients experience low sleep efficiency, increasing light sleep, and frequent arousals which all contribute to poor quality of sleep and consequently may impact all aspects of their lives.

Using the PSQI questionnaire, cases were divided into good sleepers (score less than or equal to 5 ) which represented $30 \%$ of our studied population and poor sleepers (score more than 5) which represented 70\%; the comparison between both groups showed difference as regards the duration of illness being longer in poor sleepers, and serum creatinine was found to 
be higher in good sleepers. Serum creatinine level reflects muscle mass and nutritional status; high levels indicate good nutritional status which improves sleep quality as mentioned by Ongan and Yuksel's study finding similar results regarding serum creatinine level where good sleepers had significantly higher creatinine levels than poor sleepers and the risk of poor sleep quality was lower with the increase in serum creatinine levels [46]. Also, Sabbatini et al. reported a significantly higher risk of insomnia in patients with $>12$ months on dialysis and higher levels of serum creatinine in the control group vs the insomnia group; they attributed the increased creatinine to the greater number of males and to high intake of protein [47], unlike Iliescu et al. who found no statistically significant relationship between the global PSQI score and serum creatinine level [48]. In the present study, no obvious relationship between anemia and sleep disorders which was reported also by Sabbatini et al.s study [47] unlikely other studies proved a strong correlation between both [49]. The present study also showed no relationship between other biochemical tests and sleep disorders.

Polysomnography showed a significant difference between good and poor sleepers as regards sleep efficiency $(80.4 \%$ in good sleepers vs $62.9 \%$ in poor sleepers); stage 1 of sleep was longer in poor sleepers with a shorter REM onset latency. Hence, the questionnaire can detect the presence of sleep problems as the subjects in our sample classified as poor sleepers are the cases who experience OSA or PLMD, but the questionnaire cannot diagnose OSA or PLMD. Although the several prediction models and questionnaires have been proposed for diagnosing OSA, the sensitivity of these questionnaires ranges from 80 to $90 \%$, and specificity can be as low as $34 \%$, limiting the utility of this approach [50]. So, PSG remains the criterion standard for the diagnosis of OSA [51].

In hemodialysis patients, PLMS is associated independently with increased estimated cardiovascular and cerebrovascular risk [52] and has been shown to increase the mortality risk [53]. Additionally, cardiac activation is significantly greater when leg movements are associated with obstructive sleep apnea episodes, compared to respiratory events not associated with leg movements [54], and both PLMS and OSA have been associated with daytime sleepiness, impaired cognitive function, and poor vocational abilities. Thus, identification and correction may have the potential to improve the quality of life and clinical outcomes of ESRD patients by reducing those complications associated with sleep disorders.

\section{Conclusions}

Obstructive sleep apnea in addition to PLM is common in ESRD patients proved by polysomnography, affecting their sleep quality and increasing daytime sleepiness. These findings should be disseminated among health care professionals in most medical specialties to raise their awareness and provide them with training to help with case identification and initiation of early intervention to foster the well-being of these patients.

\section{Limitation}

The study included a small number of patients hence cannot be generalized. Cases groups have HTN and DM which can be confounding factors that may affect sleep quality. Also, in the current study, we did not use any screening questionnaire to exclude any comorbid psychiatric symptoms.

\section{Abbreviations}

ESRD: End-stage renal disease; GFR: Glomerular filtration rate; HD: Hemodialysis; OSA: Obstructive sleep apnea; BMI: Body mass index; PSQI: Pittsburgh Sleep Quality Index; PLMI: Periodic Limb Movement Index; REM: Rapid eye movement.

\section{Acknowledgements}

Not applicable.

\section{Authors' contributions}

TA designed the work and reviewed the whole study findings. IS analyzed and interpreted the patient data regarding the dialysis. AM collected the data of polysomnography done to the patients and analyzed them. $\mathrm{RH}$ analyzed and interpreted the patient data regarding the sleep study and scale and dialysis and was a major contributor in writing the manuscript. All authors read and approved the final manuscript.

\section{Funding}

This research did not receive any specific grant from funding agencies in the public, commercial, or not-for-profit sectors.

\section{Availability of data and materials}

All data generated or analyzed during this study are included in this published article.

\section{Declarations}

\section{Ethics approval and consent to participate}

The procedure of the study and the design were accepted and validated by the ethical committee of Ain Shams University, Cairo, Egypt, and informed consent was given by the participants.

\section{Consent for publication}

The participants gave consent for using their data in publication.

\section{Competing interests}

The authors declare that they have no competing interests.

\section{Author details}

${ }^{1}$ Okasha Institute of Psychiatry, Faculty of Medicine, Ain Shams University, Cairo, Egypt. ${ }^{2}$ Internal Medicine and Nephrology Department, Faculty of Medicine, Ain Shams University, Cairo, Egypt. 
Received: 6 October 2021 Accepted: 26 November 2021

Published online: 10 January 2022

\section{References}

1. Cy H, Lin F, Vittinghoff E et al (2003) Racial differences in the progression from chronic renal insufficiency to end-stage renal disease in the United States. J Am Soc Nephrol 14:2902-2907

2. Collins AJ, Foley RN, Chavers B et al (2014) US Renal Data System 2013 Annual Data Report. Am J Kidney Dis 63(1 Suppl):A7

3. Steenkamp R, Castledine C, Feest T et al (2011) UK Renal Registry 13th Annual Report (December 2010): Chapter 2: UK RRT prevalence in 2009: national and centre-specific analyses. Nephron Clin Pract 119(suppl 2): $c 27-c 52$

4. Alsuwaida AO, Farag YM, Al Sayyari AA et al (2010) Epidemiology of chronic kidney disease in the Kingdom of Saudi Arabia (SEEK-Saudi investigators) a pilot study. Saudi J Kidney Dis Transpl 21:1066-1072

5. Afifi A.(2008) Egyptian renal registry $9^{\text {th }}$ Annual Reports. Available at: http://www.esnonline.net.

6. van der Tol A, Lameire N, Morton RL et al (2019) An international analysis of dialysis services reimbursement. Clin J Am Soc Nephrol 14(1):84-93. https://doi.org/10.2215/CJN.08150718 Epub 2018 Dec 13. PMID: 30545819: PMCID: PMC6364535

7. Levin A, Tonelli M, Bonventre J et al (2017) ISN Global Kidney Health Summit participants: global kidney health 2017 and beyond: a roadmap for closing gaps in care, research, and policy. Lancet 390:1888-1917

8. Vanholder R, Lameire N, Annemans L et al (2016) Cost of renal replacement: how to help as many as possible while keeping expenses reasonable? Nephrol Dial Transplant 31:1251-1261

9. Iyanage T, Ninomiya T, Jha $V$ et al (2015) Worldwide access to treatment for end-stage kidney disease: a systematic review. Lancet. 385:1975-1982

10. Mollaoğlu M. (2011) Sleep in patients with ESRD undergoing hemodialysis, progress in hemodialysis- from emergent biotechnology to clinical practice, Angelo Carpi. ISBN: 978-953-307-377- 4

11. Maung SC, El Sara A, Chapman C et al (2016) Sleep disorders and chronic kidney disease. World J Nephrol 5(3):224-232

12. Knezevic MZ, Djordjevic WV, Jankovic SM et al (2013) Influence of dialysis modality and membrane flux on insomnia severity in haemodialysis patients. Nephrology (Carlton) 18(11):706-711

13. Merlino G, Piani A, Dolso P et al (2006) Sleep disorders in patients with end-stage renal disease undergoing dialysis therapy. Nephrol Dial Transplant 21:184-190

14. Perl J, Unruh ML, Chan CT (2006) Sleep disorders in end-stage renal disease: 'markers of inadequate dialysis'? Kidney Int 70(10):1687-1693. https://doi.org/10.1038/sj.ki.5001791 Epub 2006 Sep 13.PMID: 16969388

15. Abuyassin B, Sharma K, Ayas NT, Laher I (2015) Obstructive sleep apnea and kidney disease: a potential bidirectional relationship? J Clin Sleep Med 11(8):915-924. https://doi.org/10.5664/jcsm.4946 PMID: 25845900; PMCID: PMC4513269

16. Kraus MA, Hamburger RJ (1997) Sleep apnea in renal failure. Adv Perit Dial 13:88-92

17. Fleischmann G, Fillafer G, Matterer $\mathrm{H}$ et al (2010) Prevalence of chronic kidney disease in patients with suspected sleep apnoea. Nephrol Dial Transplant 25:181-186

18. Peppard PE, Young T, Palta M et al (2000) Prospective study of the association between sleep-disordered breathing and hypertension. N Engl J Med 342:1378-1384

19. Peker Y, Carlson J, Hedner J (2006) Increased incidence of coronary artery disease in sleep apnoea: a long-term follow-up. Eur Respir J 28:596-602

20. Punjabi NM, Polotsky VY (2005) Disorders of glucose metabolism in sleep apnea. J Appl Physiol 99:1998-2007

21. Ogna A, Forni Ogna V, Mihalache A et al (2015) Obstructive sleep apnea severity and overnight body fluid shift before and after hemodialysis. Clin J Am Soc Nephrol 10(6):1002-1010. https://doi.org/10.2215/CJN.08760 914 Epub 2015 Apr 10. PMID: 25862778; PMCID: PMC4455216

22. United States Renal Data System (2015) USRDS Annual Data Report: epidemiology of kidney disease in the United States. National Institutes of Health, National Institute of Diabetes and Digestive and Kidney Diseases, Bethesda
23. Park Jl, Bae E, Kim YL et al (2015) Glycemic control and mortality in diabetic patients undergoing dialysis focusing on the effects of age and dialysis type: a prospective cohort study in Korea. PLoS One 10:e0136085

24. Yi C, Guo Q, Lin J et al (2016) Clinical outcomes of remote peritoneal dialysis patients: a retrospective cohort study from a single center in China. Blood Purif 41:100-107

25. Buysse DJ, Reynolds CF 3rd, Monk TM et al (1989) The Pittsburgh Sleep Quality Index: a new instrument for psychiatric practice and research. Psychiatry Res 28(2):193-313

26. Asaad T, Kahla A (2009) Sleep problems and diagnosis. Egypt: Dar Eirtak for Printing, Publishing and Distribution Co., 169-383-977, p. 22-25

27. Ohayon MM, Carskadon MA, Guilleminault C, Vitiello MV (2004) Metaanalysis of quantitative sleep parameters from childhood to old age in healthy individuals: developing normative sleep values across the human lifespan. Sleep 1;27(7):1255-73. https://doi.org/10.1093/sleep/ 27.7.1255

28. Bonnet M, Arand D (2007) EEG Arousal Norms by Age. J Clin Sleep Med 3(3):271-274

29. Recording and scoring leg movements (1993) The Atlas Task Force. Sleep 16:748-759

30. Peters B. (2011) Definition of AHI. Available from http://sleepdisorders. about.com/od/glossary/g/AHI.htm

31. Goyal M, Johnson J (2017) Obstructive Sleep Apnea Diagnosis and Management. Missouri Med 114(2):120-24

32. American Academy of Sleep Medicine (2005) International Classification of Sleep Disorders. Diagnostic and Coding Manual, 1st edn. American Academy of Sleep Medicine, Westchester

33. Hanly P (2004) Sleep apnea and daytime sleepiness in end-stage renal disease. Semin Dial 17:109-114

34. Beecroft JM, Pierratos A, Hanly PJ (2009) Clinical presentation of obstructive sleep apnea in patients with end-stage renal disease. J Clin Sleep Med 5:115-121

35. Ezzat H, Mohab A (2015) Prevalence of sleep disorders among ESRD patients. Ren Fail 37(6):1013-1019. https://doi.org/10.3109/0886022X. 2015.104440

36. Nicholl DD, Ahmed SB, Loewen AH et al (2012) Declining kidney function increases the prevalence of sleep apnea and nocturnal hypoxia. Chest 141:1422-1430

37. Kuhlmann U, Becker HF, Birkhahn M et al (2000) Sleep-apnea in patients with end-stage renal disease and objective results. Clin Nephrol 53(6):460-466

38. Beecroft JM, Zaltzman J, Prasad GV et al (2008) Improvement of periodic limb movements following kidney transplantation. Nephron Clin Pract 109:c133-c139

39. Jurado-Gamez B, Martin-Malo A, Alvarez-Lara MA et al (2007) Sleep disorders are underdiagnosed in patients on maintenance hemodialysis. Nephron Clin Pract 105:c35-c42

40. Hanly PJ, Gabor JY, Chan C et al (2003) Daytime sleepiness in patients with CRF: impact of nocturnal hemodialysis. Am J Kidney Dis 41:403-410

41. Rijsman RM, de Weerd AW, Stam CJ et al (2004) Periodic limb movement disorder and restless legs syndrome in dialysis patients. Nephrology (Carlton) 9(6):353-361. https://doi.org/10.1111/j.1440-1797.2004.00330.x PMID: 15663636

42. Huang CY, Yu CC (2019) Different diagnostic criteria for periodic leg movements in patients with obstructive sleep apnea after continuous positive airway pressure titration. Neuropsychiatr Dis Treat 15:2129-2136. Published 2019 Jul 24. https://doi.org/10.2147/NDT.S209806

43. Shrivastava D, Jung S, Saadat M et al (2014) How to interpret the results of a sleep study. J Community Hosp Intern Med Perspect 4(5):24983. Published 2014 Nov 25. https://doi.org/10.3402/jchimp.v4.24983

44. BaHammam AS, Al-Shimemeri SA, Salama Rl et al (2013) Clinical and polysomnographic characteristics and response to continuous positive airway pressure therapy in obstructive sleep apnea patients with nightmares. Sleep Med 14:149-154. https://doi.org/10.1016/j.sleep.2012.07.007

45. Roumelioti ME, Buysse DJ, Sanders MH et al (2011) Sleep-disordered breathing and excessive daytime sleepiness in chronic kidney disease and hemodialysis. Clin J Am Soc Nephrol 6:986-994 CrossrefMedlineGoogle Scholar/0886022X.2015.1044401. Epub 2015 May 11. PMID: 25959021 
46. Ongan D, Yuksel A (2017) What to eat for a better sleep in haemodialysis patients: potential role of B vitamins intake and appetite. Pakistan J Med Sci 33(2):417-424. https://doi.org/10.12669/pjms.332.11838

47. Sabbatini M, Minale B, Crispo A et al (2002) Insomnia in maintenance haemodialysis patients. Nephrol Dial Transplant 17(5):852-856. https:// doi.org/10.1093/ndt/17.5.852 PMID: 11981073

48. Iliescu EA, Yeates KE, Holland DC (2004) Quality of sleep in patients with chronic kidney disease. Nephrol Dial Transplant 19(1):95-99. https://doi. org/10.1093/ndt/gfg423

49. Sabry AA, Abo-Zenah H, Wafa E et al (2010) Sleep disorders in hemodialysis patients. Saudi J Kidney Dis Transpl 21(2):300-305 PMID: 20228517

50. Rowley JA, Aboussouan LS, Badr MS (2000) The use of clinical prediction formulas in the evaluation of obstructive sleep apnea. Sleep. 23:929-938

51. National Government Services, Inc (2010). LCD for positive airway pressure (PAP) devices for the treatment of obstructive sleep apnea (L27230): American Medical Association; Center for Medicaid and Medicare. http:// www.nationwidemedical.com/wp-content/uploads/2010/06/LCD-forPositive-Airway-Pressure-doc-region-b.pdf Accessed 22 Mar 2011.

52. Lindner A, Fornadi K, Lazar AS et al (2012) Periodic limb movements in sleep are associated with stroke and cardiovascular risk factors in patients with renal failure. J Sleep Res 21(3):297-307. https://doi.org/10.1111/j. 1365-2869.2011.00956

53. Yumino D, Wang H, Floras JS et al (2011) Relation of periodic leg movements during sleep and mortality in patients with systolic heart failure. Am J Cardiol 107:447-451. https://doi.org/10.1016/j.amjcard.2010.09.037

54. Yang CK, Jordan AS, White DP et al (2006) Heart rate response to respiratory events with or without leg movements. Sleep 29:553-556

\section{Publisher's Note}

Springer Nature remains neutral with regard to jurisdictional claims in published maps and institutional affiliations.

\section{Submit your manuscript to a SpringerOpen ${ }^{\circ}$ journal and benefit from:}

- Convenient online submission

- Rigorous peer review

- Open access: articles freely available online

- High visibility within the field

- Retaining the copyright to your article

Submit your next manuscript at $\boldsymbol{\nabla}$ springeropen.com 